\title{
BALANCE DEL PLAN DE MODERNIZACIÓN DE LOS SERVICIOS INTERURBANOS POR AUTOBÚS
}

\author{
Montserrat Andújar Martínez \\ Consorcio Regional de Transportes de Madrid \\ Elena Tarruella Vidal \\ Consorcio Regional de Transportes de Madrid \\ Berta Nieto Corella \\ Consorcio Regional de Transportes de Madrid \\ María del Amor León Fariñas \\ Consorcio Regional de Transportes de Madrid
}

\section{RESUMEN}

El Plan de Modernización de los servicios de transporte público regular permanente de viajeros del Consorcio Regional de Transportes de Madrid, aprobado a finales de 2009, tiene como objetivo revitalizar el servicio de transporte en los autobuses urbanos e interurbanos de la Comunidad de Madrid.

Este Plan abarca tanto a los vehículos como a la tecnología necesaria para la mejora del servicio y de la información al usuario. Las mejoras llevadas a cabo han implicado unos desarrollos e inversiones cuantiosos como la instalación de los SAEs, implantación del sistema de ticketing sin contacto, instalación de displays embarcados, cámaras de videovigilancia, información en tiempo real a través de diferentes soportes (Pantallas en paradas o intercambiadores, Aplicaciones móviles,...), adaptación de los vehículos para mejorar la accesibilidad a personas de movilidad reducida,...

Tras seis años de intenso trabajo, tanto por parte de la Administración como de las empresas operadoras, se han alcanzado unos estándares elevados en la totalidad de los aspectos, habiéndose cumplido los objetivos perseguidos, con carácter general, como demuestran los resultados de las encuestas de satisfacción a los usuarios.

Además, dentro de este marco, en los últimos años, se han ido desarrollando nuevos aspectos tecnológicos, que serán fundamentales para el buen desarrollo de las labores del Consorcio, como son la integración de la información en tiempo real de todos los operadores en el CITRAM, la utilización de las herramientas de gestión de incidencias y afecciones, etc. 


\section{ANTECEDENTES}

En 2009, en la Comunidad de Madrid el servicio regular de transporte de viajeros por carretera se prestaba mediante diversas concesiones, repartidas a lo largo de los distintos corredores radiales, fundamentalmente.

Desde la creación del Consorcio Regional de Transportes en 1986, se había pasado de gestionar 33 líneas urbanas distribuidas en 14 municipios a 127 líneas en 33 municipios en el año 2009, mientras que las líneas interurbanas se incrementaron desde 144 en 1986 a 346 en 2009.

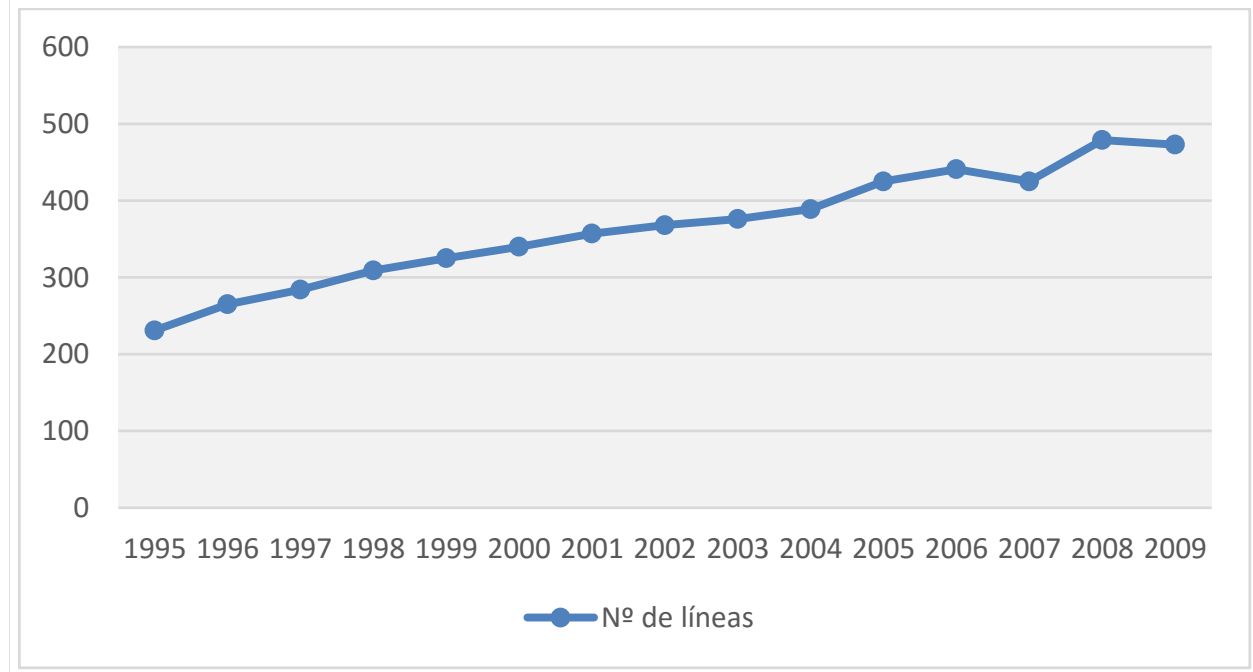

Fig. 1 - Evolución de la red (líneas urbanas e interurbanas)

Este aumento continuado de la oferta de servicio, sin embargo, no había ido acompañado, sobre todo en los últimos años, de un incremento de demanda, sino más bien al contrario, por lo que los niveles de subvención al sistema de transporte interurbano se habían incrementado considerablemente.

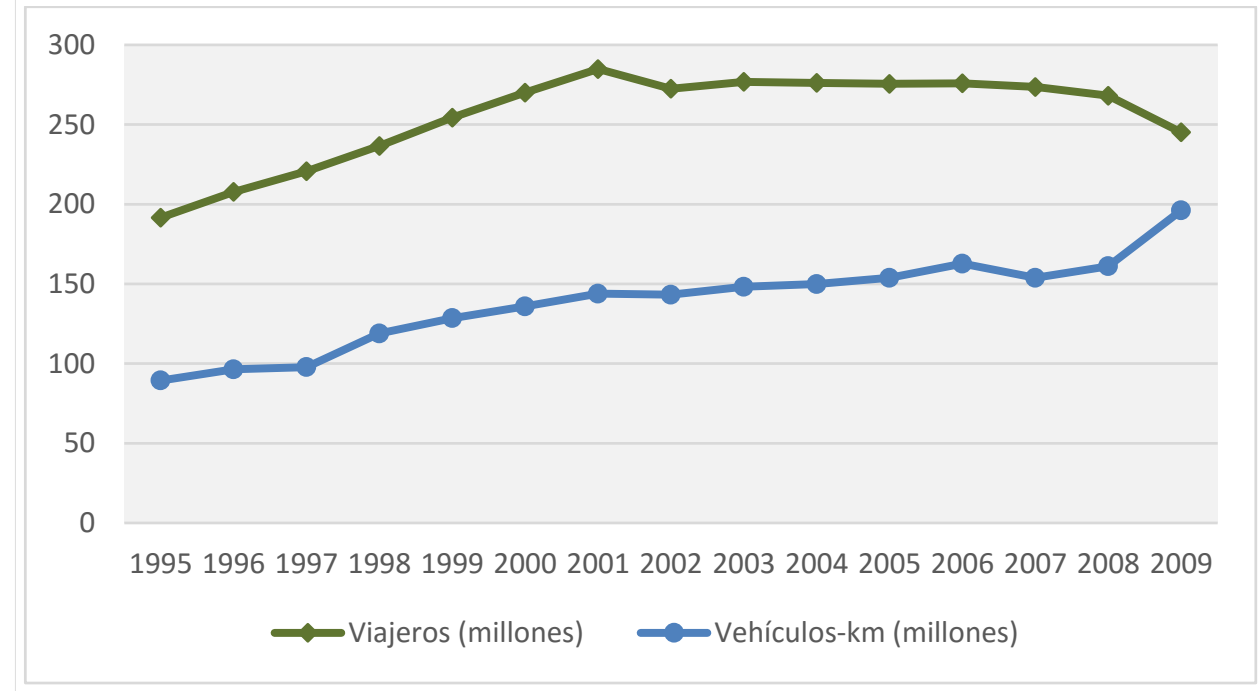




\section{Fig. 2 -Relación entre la oferta y la demanda}

\section{OBJETIVOS DEL PLAN DE MODERNIZACIÓN}

En la Ley 5/2009 de Ordenación del Transporte y la Movilidad por Carretera de la Comunidad de Madrid, se define el contenido y exigencias del Plan de Modernización. Los objetivos principales son:

1. Mejorar la calidad del servicio.

2. Mejorar la calidad de la información al usuario.

3. Disminuir la antigüedad de la flota.

4. Mejorar la accesibilidad a la red de autobuses interurbanos.

El Plan de Modernización se establece en el marco del proceso de convalidación de las concesiones existentes, aprobado por la Asamblea Regional de la Comunidad de Madrid, las cuales podían optar por mantener sus contratos concesionales hasta su extinción, o sustituir los mismos por otros de 10 años de duración, siempre que acometiesen diversos requerimientos contenidos en el Plan de Modernización.

\section{CONTENIDO Y AVANCES}

Los objetivos anteriormente mencionados se concretan en el Plan mediante unas medidas específicas que habrían de adoptar los operadores que se adhiriesen al mismo. También, para cada una de estas medidas, se indican los plazos en los que completar cada una de esas actuaciones. A continuación se hace un análisis del contenido de cada una de estas medidas.

\subsection{Plan de Calidad}

El Plan de Calidad tiene diversos objetivos: implantar un modelo de calidad de cumplimiento para todos los operadores, para promover la eficiencia y potenciar la utilización del sistema de transporte público; establecer unos criterios de referencia homogéneos; introducir elementos de evaluación adecuados y suficientes para que el modelo de calidad se pueda mantener en el tiempo y evolucionar, si fuese necesario; impulsar los procesos de certificación y regular la repercusión económica de los requisitos exigidos en la gestión de los servicios.

El Plan de Calidad definido se basa en la norma UNE EN-13816, en la que se establecen unos indicadores de calidad que se adaptaron a las circunstancias y especificidades del transporte público para poder establecer tanto los objetivos y alcance de los mismos, como las metodologías de medición, así como el baremo de bonificaciones o penalizaciones según su cumplimiento.

Los indicadores se estructuran en ocho grupos:

1. Servicio ofertado 
2. Accesibilidad

3. Información al usuario

4. Confort

5. Seguridad

6. Impacto Ambiental

7. Atención al cliente

8. Valoración del usuario

Dentro de la variedad de indicadores definidos, se pretendió que la mayoría fuesen fácilmente medibles para su adecuada valoración cuantitativa, aunque en algunos aspectos era inevitable la consideración cualitativa de los mismos, como en el caso del indicador relativo a la "Valoración del usuario", indicador que se obtiene mediante encuestas de satisfacción supervisadas por el CRTM.

Dentro de la filosofía del Plan, se han desarrollado, por parte del CRTM, ciertas herramientas que permiten la efectiva medición de algunos indicadores como es la aplicación GEIS (Gestión de incidencias). Mediante esta aplicación, los operadores han de informar al CRTM de todas aquellas incidencias y afecciones que se produzcan en sus líneas en tiempo real, o al menos en un tiempo razonablemente corto desde que se produzca, dando prioridad en todo caso, a las labores que tuviesen que realizar de aviso a cuerpos de seguridad o asistencia sanitaria, en su caso, y de comunicación a sus conductores de afecciones al servicio, si fuese necesario.

Además, en el Plan de Calidad, se establecía que si el operador obtenía la certificación UNE EN-13816, obtendría una bonificación adicional, siempre que el resultado de aplicar el Plan de Calidad fuese positivo o neutro. Esto ha significado que los operadores han ido adaptando sus servicios para cumplir con esta norma y, en la actualidad, todas las concesiones han conseguido esta certificación.

\subsection{Medidas tecnológicas}

El aspecto más novedoso que ha permitido implantar el Plan de Modernización es la integración de las nuevas tecnologías en todas las concesiones del CRTM. Debido a la diversidad de operadores y de cómo se habían ido gestionando cada una de ellas, la situación de partida era muy diferente de unos a otros. Así se estableció una batería de medidas, para conseguir unos altos estándares tecnológicos, que ha facilitado una mejora importante en la gestión y explotación del servicio.

\subsubsection{Sistema de ayuda a la explotación (SAE)}

Implantar un Sistema de Ayuda a la Explotación (SAE) era fundamental para la puesta en marcha de cualquier medida posterior que se quisiese desarrollar. Por este motivo, se establecieron unos estándares generales para que cada concesión adaptara la solución que estimara más interesante, pudiendo incorporar, si así lo consideraba necesario, otras 
utilidades que les pudiesen resultar necesarias.

Todas las empresas operadoras han instalado su SAE correspondiente, que permite tanto la localización de los vehículos como la identificación de la línea que están realizando, en tiempo real, así como la obtención de datos de explotación, que son utilizados tanto en estudios de análisis de grado de cumplimiento, como de ayuda para el análisis de las quejas y sugerencias recibidas en el CRTM.

\subsubsection{Sistemas de Billetaje sin contacto}

Actualmente, todos los Abonos Transporte de la Comunidad de Madrid, utilizan la tecnología sin contacto (BIT), aunque ya desde el 2012 se había ido implantando paulatinamente, primero en la corona tarifaria A (desde mayo 2012) y, posteriormente, en el resto de zonas tarifarias.

Con el objetivo de que estos títulos pudiesen ser utilizados en todo el sistema de transporte, se realizó la implantación del hardware y software definido por el CRTM para la validación a bordo de los mismos y su adecuada identificación, en todos los vehículos de la flota de los servicios interurbanos y urbanos de las zonas B y C.

La versatilidad de esta tecnología permite incorporar nuevos títulos al sistema, lo que favorece la agilidad de su implantación. Este ha sido el caso del último tipo de Abono incorporado en el sistema: el Abono Joven hasta los 26 años con una tarifa plana, para toda la Comunidad de Madrid y zonas E1 y E2, de $20 €$, que ha sido un éxito en su aceptación por parte de los jóvenes.

\subsubsection{Comunicaciones inalámbricas}

Se establece como exigencia que los autobuses dispusiesen de sistemas de comunicaciones inalámbricos, que permitiesen la comunicación de los datos de explotación y de los sistemas de billetaje, para lo que el CRTM estableció protocolos y sistemas de seguridad determinados.

Este requisito también está implantado en todos los operadores y, de forma diaria, envían al CRTM a través de unos archivos codificados las validaciones de los títulos sin contacto. Además el resto de información es codificada y guardada para el posterior envío al CRTM en los formatos definidos al efecto. Asimismo estas comunicaciones, ya sean a través de Wi-Fi, GPRS, 3G,..., permiten a los vehículos conectarse con el Centro de Control Local, para su supervisión y obtención de la información necesaria.

\subsubsection{Sistema dinámico de información al usuario}

Otro de los puntos clave del Plan de Modernización es la mejora de la información al usuario, fundamentalmente en tiempo real, teniendo en cuenta, además, los diferentes tipos de usuarios y sus capacidades. 
Así se han instalado en el interior de todos los vehículos, sistemas de información de próxima parada, mediante displays a bordo, en los que se informa mediante información visual y acústica.

Además para mejorar la información en tiempo real en las paradas, se han instalado Paneles de Información Variable (PIV) en los puntos más importantes identificados en cada uno de los municipios y, pantallas de información en algunos Hospitales de la Comunidad. Este aspecto ha sido unos de los mejor valorados por los usuarios en los últimos años como mejora del sistema de transporte, pues permite saber la hora de paso por la parada de la línea correspondiente y hace que la espera del usuario se perciba de forma más atractiva. En la actualidad existen 449 PIV instalados en 362 paradas distribuidos en distintos puntos de la Región.

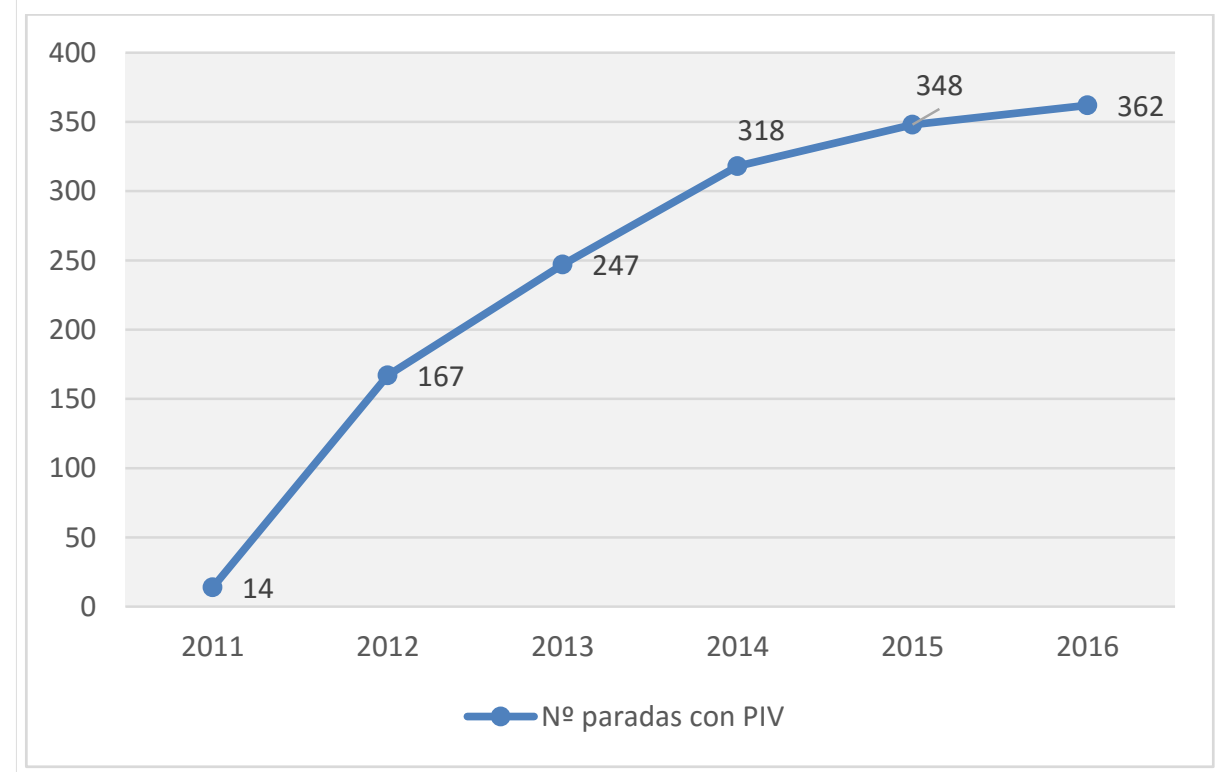

Fig. 3 -Paradas con Paneles de Información Variable (PIV) instalados.

Otras de las herramientas que se han desarrollado dentro del Plan para facilitar la información en tiempo real a los usuarios han sido tanto el desarrollo de la App Mi transporte, como del acceso Web desde la página del CRTM.

La App Mi transporte, proporciona información en tiempo real de todos los modos de transporte de la Comunidad de Madrid, facilitando la hora de paso por parada de los distintos servicios, eligiendo una parada concreta según la ubicación del usuario. La aplicación ha tenido una gran aceptación entre los usuarios y está disponible tanto para el sistema Android como para Apple. El desarrollo de esta aplicación, que ofrece información integrada de cuarenta operadores de transporte, ha supuesto uno de los retos tecnológicos más exigente del Plan de Modernización.

En cuanto al acceso de la información en tiempo real desde la Web, es de implantación 
muy reciente, pues ha sido en abril de este año cuando se ha puesto en marcha.

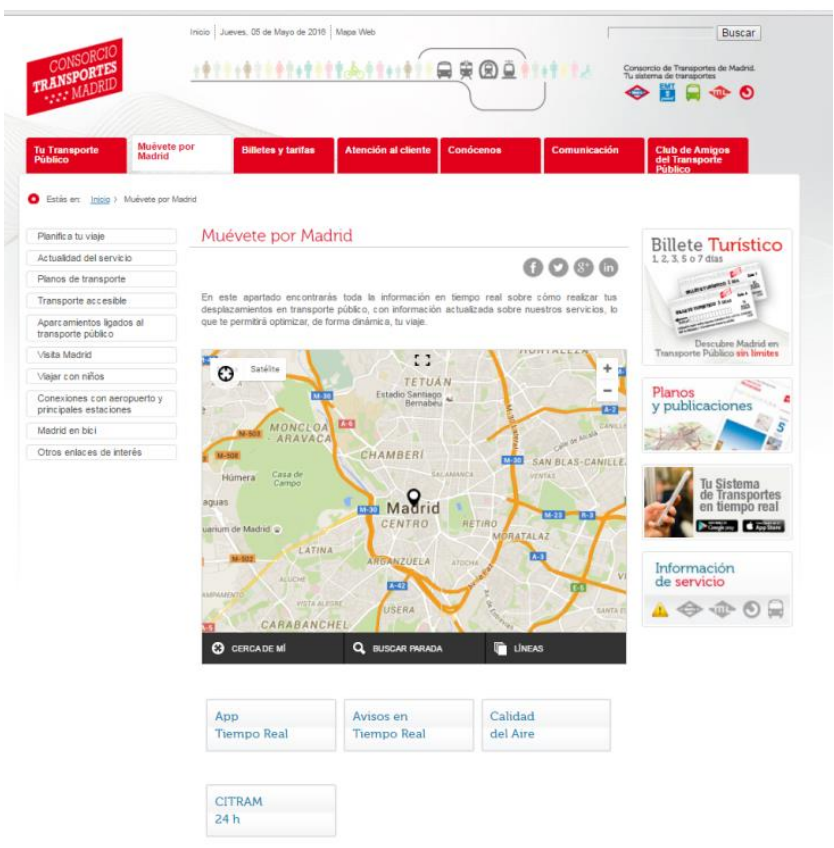

Fig. 4 -Web del CRTM de acceso a la información en tiempo real.

\subsubsection{Centro de Control Local (CCL)}

Todas las empresas operadoras han instalado un Centro de Control Local (CCL) en sus instalaciones que incorpora los sistemas, hardware y software necesarios para supervisar los vehículos en servicio, en cada una de las líneas en tiempo real, así como la trasmisión de los datos al CRTM. Asimismo estos CCL almacenan la información de explotación, para que posteriormente pueda ser utilizada por los servicios técnicos del operador y/o del CRTM.

\subsubsection{Cámaras de vigilancia (CCTV)}

Aunque el Plan exigía sólo que los autobuses que prestaran servicios nocturnos tuviesen instaladas cámaras de seguridad, conectadas con una Central de Alarmas, son varias las empresas que han optado por incorporar dicha funcionalidad en el total de la flota.

En la actualidad, se está desarrollando un sistema de modo para que pueda ser el propio CRTM, desde su Centro de Gestión Multimodal, el que visualice las cámaras de manera integrada, sin necesidad de emplear el cliente de visualización de uno u otro operador.

\subsubsection{Actualización de información dinámica y estática}

El CRTM desarrolló una aplicación Web (GIW) para facilitar el ajuste y modificación de horarios e itinerarios de una forma más eficiente, y así poder relacionar la base de datos existente en el Consorcio, basada en ORACLE, con los sistemas SAE de cada uno de los operadores. 
Así a través de esta aplicación se reciben todas las solicitudes de modificación mediante propuestas de horarios o itinerarios, que tras ser analizadas en el Consorcio, si son adecuadas, se procede a su aceptación. Esta aceptación implica su incorporación inmediata en la base de datos del CRTM, lo que supone una mejora sustancial en el desarrollo de estas tareas, alcanzando unos niveles de eficiencia elevados.

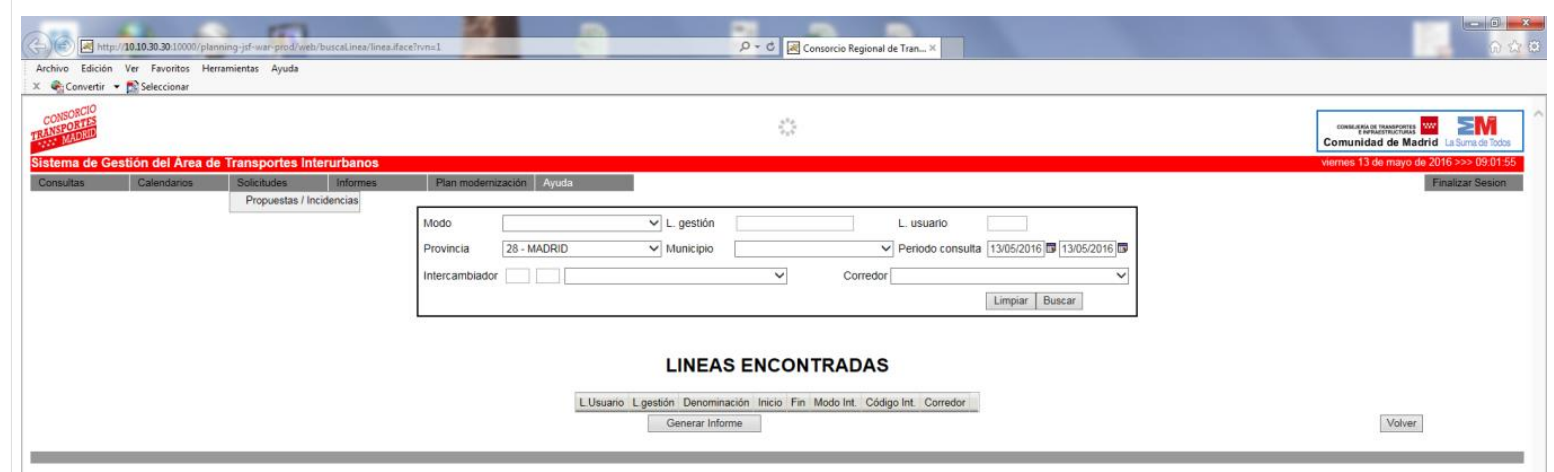

Fig. 5 -Aplicación de gestión de modificación de itinerarios y horarios (GIW).

Además, esta aplicación es utilizada para obtener por parte de los operadores otras informaciones necesarias para el buen funcionamiento de los sistemas de intercambio de información, como es la información de topología de la red, mediante archivos XML, la información sobre los calendarios horarios vigentes según el tipo de día;...

\subsection{Mejoras en la flota}

La renovación de la flota era uno de los objetivos más importantes del Plan de Modernización, debido a que, en los años anteriores a la publicación del mismo, el envejecimiento de la misma había deteriorado su imagen, en contra de los estándares de eficiencia medioambiental y mejora de la accesibilidad que demandaba la sociedad.

Las medidas que se exigían en este aspecto eran: Reducir la edad máxima de los vehículos de 12 a 10 años; alcanzar una edad máxima de la flota no superior a 5 años y garantizar la accesibilidad a personas con movilidad reducida en los vehículos.

En la fecha prevista, el total de los operadores cumplieron estos objetivos de edad máxima, edad media y accesibilidad. Las inversiones que cada uno de los operadores hubieron de hacer para lograr estos objetivos fueron cuantiosas, teniendo en cuenta que en dos años se renovaron 620 vehículos, lo que supone el $29 \%$ de la flota. En los siguientes años se ha continuado con la renovación de los mismos para que estas condiciones se mantuviesen, tal como indica el Plan de Modernización, llegando a renovar 1.309 vehículos hasta 2015. 


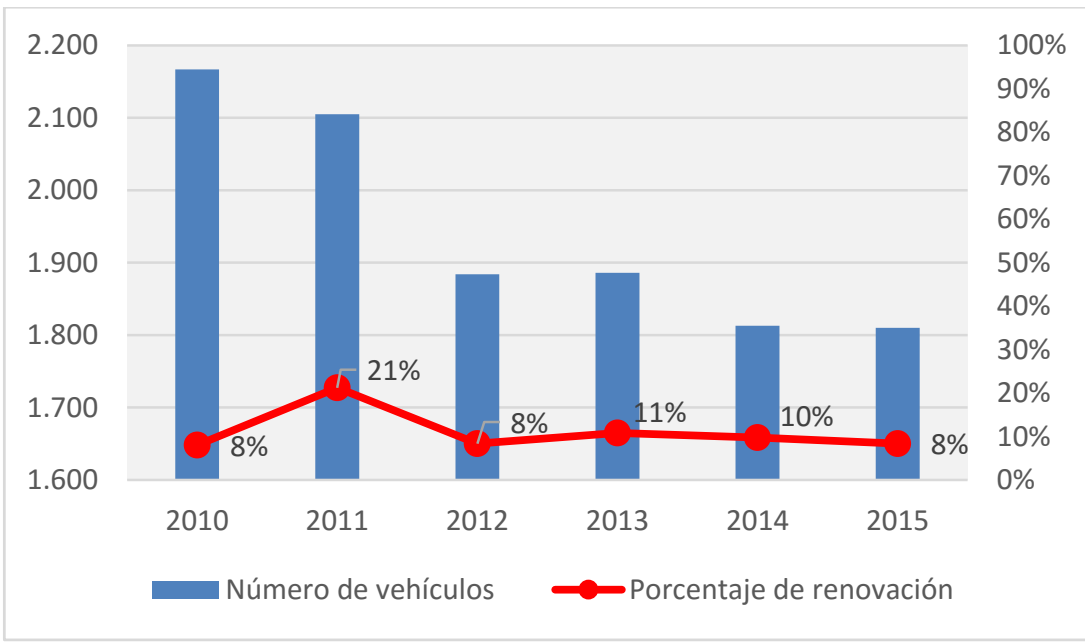

Fig. 6 -Renovación de la flota.

Como consecuencia de estas inversiones en nuevos vehículos, las empresas han sido capaces de mantener la edad media de la flota por debajo de 5 años, lo que ha supuesto una mejora en la percepción de los servicios de autobús por parte de los usuarios. Asimismo en la actualidad, el total de la flota posee rampas o plataformas elevadoras para permitir el acceso de personas de movilidad reducida, ya sea mediante sillas de ruedas u otros dispositivos de ayuda a la movilidad.

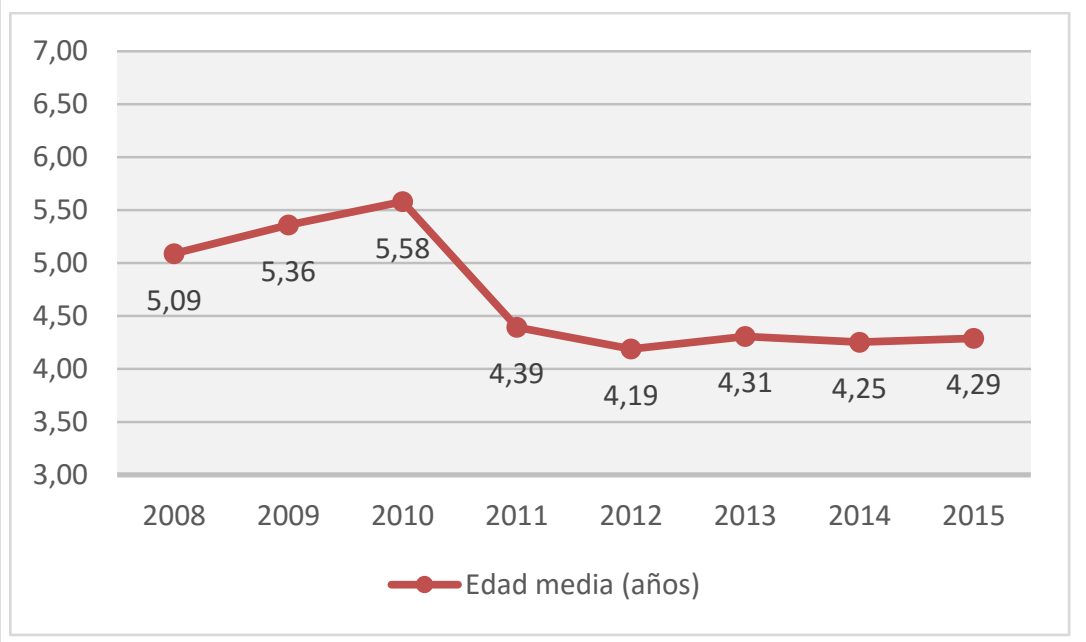

Fig. 7-Evolución de la edad media de la flota.

Otra medida adicional fue homogeneizar la señalización de los vehículos, con especial atención a la normativa de accesibilidad, para lo que el CRTM elaboró un Manual de Identidad Corporativa, de obligado cumplimiento en toda la flota de vehículos interurbanos.

\subsection{Integración de los sistemas de cada Centro de Control Local en el Centro de Gestión 24h del CRTM, CITRAM}

En el CITRAM se tiene actualmente acceso a todos los sistemas de explotación de los operadores, lo que permite supervisar permanente la explotación, así como actuar de la 
forma más ágil posible para que posibles incidencias afecten en la menor medida posible a los usuarios. Asimismo, la integración entre los sistemas de información centrales y los locales permite informar a los usuarios de cada uno de los modos de dichas perturbaciones del transporte para que puedan elegir las alternativas de movilidad más adecuadas a sus necesidades.

Este aspecto ha supuesto unas inversiones excepcionales por parte del CRTM para dotar al CITRAM de la tecnología necesaria, consiguiendo tener integrados todos los modos de transporte público en un mismo centro, proyecto pionero en Europa.

\subsection{Inversiones excepcionales}

Además de las medidas de renovación de flota indicadas anteriormente, se especificaron unas medidas excepcionales que implicaban la incorporación de tecnologías más limpias y energéticamente sostenibles tanto en la flota de los vehículos urbanos como de los interurbanos, cuyos resultados se muestran en los siguientes gráficos:

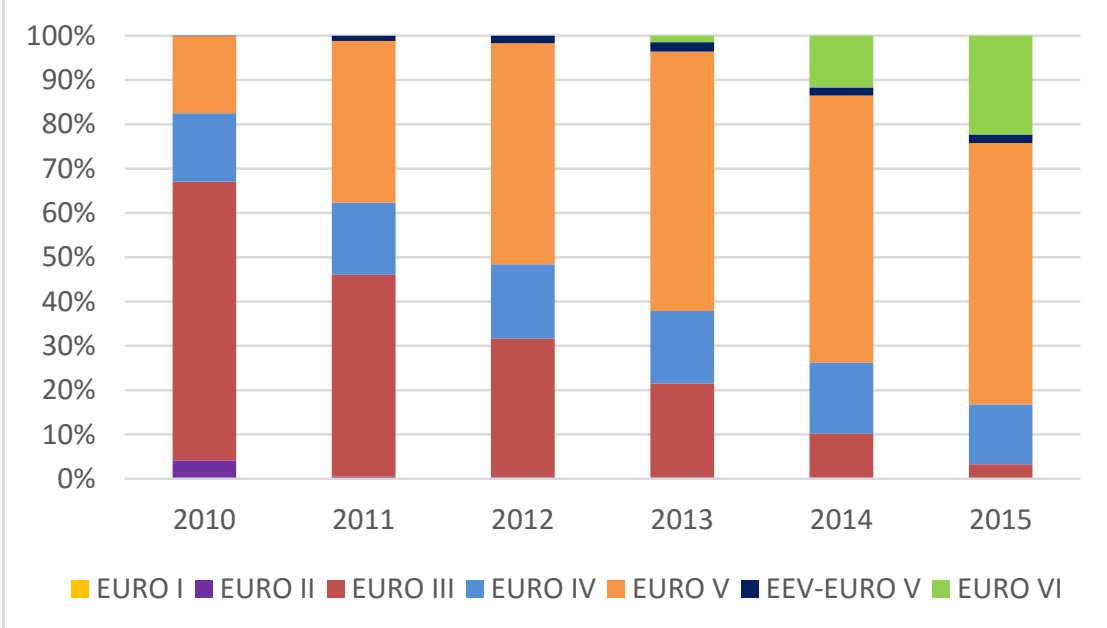

Fig. 8 - Distribución de la flota por tipo de motorización.

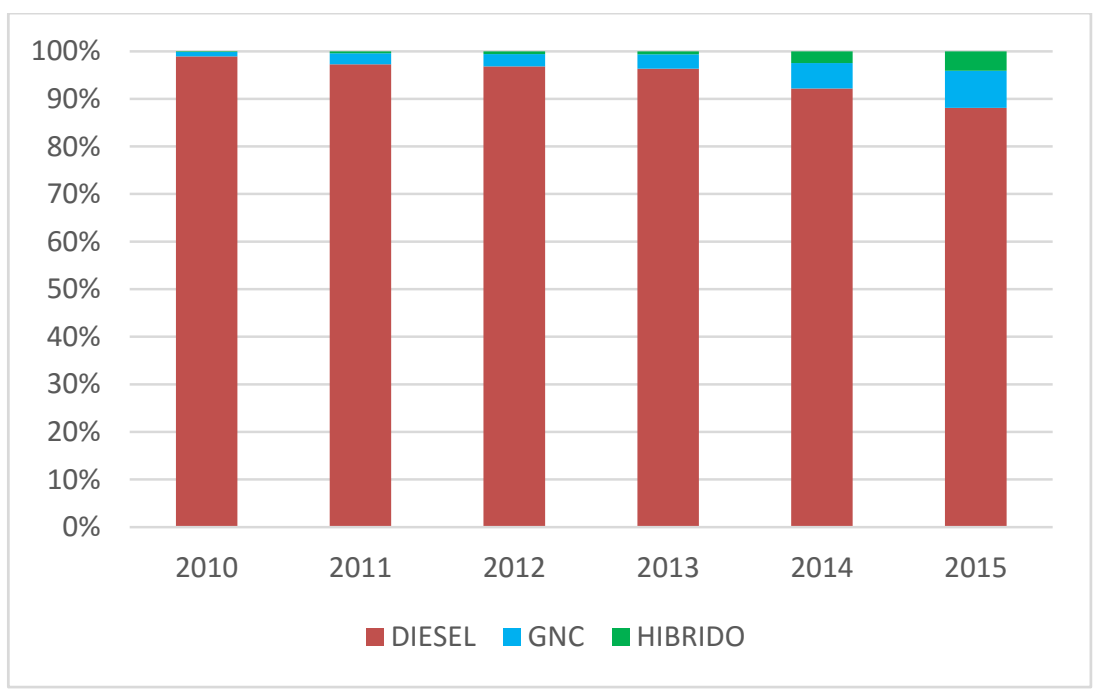




\section{Fig. 9 - Distribución de la flota por tipo de combustible.}

\subsection{Otros}

Además de todas estas medidas se definieron otras mejoras trasversales que afectan tanto a la gestión del personal como empresarial de las concesiones, entre ellas:

- Medidas socio-laborables: Planes de formación continua para los empleados, potenciación del empleo femenino, fomento de contratación de discapacitados,..

- Mejoras de gestión: Colaborar con el CRTM para modificar las líneas actuales, de forma que se puedan explotar algunas de forma conjunta, disponer de sistemas de control económico-financiero de las empresas,...

\section{CONCLUSIONES}

Tras más de cinco años de desarrollo del Plan de Modernización, con carácter general, se han cumplido sus ambiciosos objetivos, llegando incluso más allá de lo previsto inicialmente, como en el caso de la puesta en marcha de la App móvil "Mi transporte", que integra información en tiempo real de más de 450 líneas operadas por unas 30 empresas diferentes.

Gracias a todo lo desarrollado en este Plan, está previsto en este año poner a disposición de los desarrolladores un portal de datos Open Data para que cualquier interesado pueda utilizar la información.

Así el balance del Plan de Modernización es claramente positivo, habiendo contribuido a mejorar el sistema de transporte de viajeros por autobús de la Comunidad de Madrid, así como la percepción que del mismo tienen los usuarios. 